\title{
Tratamiento de la incontinencia urinaria de esfuerzo con el sistema TVT: Complicaciones en nuestros primeros 100 casos
}

\author{
C. Quicios Dorado, E. Fernández Fernández, I. Gómez García, L. Perales Cabanas, \\ F. Arias Fúnez, A. Escudero Barrilero
}

\author{
Servicio de Urología. Hospital Ramón y Cajal. España. Madrid
}

Actas Urol Esp2005; 29 (8): 750-756

\section{RESUMEN}

TRATAMIENTO DE LA INCONTINENCIA URINARIA DE ESFUERZO CON EL SISTEMA TVT: COMPLICACIONES EN NUESTROS PRIMEROS 100 CASOS

Introducción: El sistema TVT (Tension-free Vaginal Tape) es la técnica quirúrgica de elección en los últimos años de la incontinencia urinaria de esfuerzo femenina (IUE), dada la facilidad de la técnica, buenos resultados clínicos y escasas complicaciones.

Material y métodos: Desde Abril de 1999 a Marzo 2004 se han implantado en nuestro servicio 100 sistemas TVT con revisión de los resultados a los 6 meses, y un seguimiento medio de 10,36 meses (R: 1-54 meses), con una pérdida de seguimiento en el 11\% de los casos. Todas las mujeres estudiadas presentaban incontinencia de esfuerzo, siendo la edad media de 56,5 años (30-80). Un 12\% habían sido intervenidas previamente de IUE. Presentan cirugia previa del suelo pélvico 21 pacientes. En un 17\% de los casos se realizó TVT asociado a otra técnica quirúrgica: $15 \%$ cistorrafia anterior, $1 \%$ corrección de rectocele, $1 \%$ cirugía compleja del suelo pélvico que incluye TVT, corrección de cistocele e histerectomía.

Resultados: Obtenemos un 65\% de éxitos (comprobación objetiva de la ausencia de escape por parte del médico y referencia subjetiva de la paciente), un $17 \%$ de mejoría (disminución clara de escapes por parte de la paciente y mejoría subjetiva) y un $7 \%$ de fracaso de la técnica. El porcentaje global de aparición de urgencia de novo posquirúrgica es del 9\%. Existieron un $12 \%$ de complicaciones; 3 casos (3\%) de perforación vesical, 2 de ellos intraoperatorios y 1 diferida junto con infección de la herida quirúrgica; 3 episodios (3\%) de residuos altos en el postoperatorio inmediato, resueltos con autosondajes, y un caso de RAO (1\%) dado de alta con cistostomía suprapúbica; un caso (1\%) de peritonitis química tras retirada de talla vesical resuelta con tratamiento conservador; dos casos (2\%) de extrusión vaginal de la bandeleta, con sección de la porción de malla exteriorizada en ambos casos; un (1\%) paciente presenta hematoma hipogástrico que se resuelve con medidas conservadoras; un $1 \%$ de los pacientes $(1$ caso) presentó molestias pélvicas subcrónicas con resolución espontánea.

Conclusiones: Consideramos el sistema TVT una técnica quirúrgica eficaz en el tratamiento de la incontinencia urinaria femenina de esfuerzo. Es una técnica sencilla, con baja estancia hospitalaria y escasas complicaciones.

Palabras clave: Incontinencia urinaria de esfuerzo. TVT.

\section{ABSTRACT}

TRATMENT OF FEMALE STRESS URINARY INCONTINENCE WITH TVT SYSTEM (TENSION-FREE VAGINAL TAPE): COMPLICATIONS IN OUR FIRST 100 CASES.

Introduction: The TVT system is the most common surgical technique of female stress urinary incontinence, because of the simplicity, good clinical results and rare complications.

Material and methods: From april, 1999 to march, 2004, it has been done in our department 100 TVT systems with the revision of the result over the six following months. The average follow-up rate was 10, 36 months (R: 1-54 months) and looses mean the 11\% of the cases. All the patients presented stress incontinence and their average age was 56,6 years old (30-80). The $12 \%$ of the cases have been previously operated of urinary incontinence. 21 patients presented previous surgery of pelvic floor. In the $17 \%$ of the cases, TVT systems was associated to another surgical technique: $15 \%$ of them was operated of cystocele, $1 \%$ of them had surgical correction of rectocele, $1 \%$ of them had a strong surgery of pelvic floor, that included TVT system, correction of pelvic floor and hysterectomy.

Results: We obtained $65 \%$ of successful cases, defined as objective confirmation of absence of looses from the observer and the subjective reference of the patient; $17 \%$ of clear improvement (clear decrease of looses from the patient and subjective improvement) and $7 \%$ of failure of the technique. The total percentage of appearance of novo post-surgery urgency is $9 \%$. There were complications in the $12 \%$ of the cases: there were three cases of bladder perforation, two of them intra-operative and one of them deferred and associated to an infection of surgical wound; another three cases of residue in the immediate post-operative that were resolved with bladder catheterization; one case of acute urinary retention that was treated with bladder catheterization with suprapubic cistostomy; one case of chemical peritonitis corrected with a conservative treatment; two cases of vaginal extrusion of sling, which were resolved with the section of the outer sling; one patient presented a hipogastric hematoma resolved with conservative measures; and one patient presented pelvic discomfort with spontaneous resolution.

Conclusions: We consider the TVT system as an effective surgical technique in the treatment of the female stress urinary incontinence. It is a simple technique with a short stay in the hospital and rare complications.

Keywords: Stress urinary incontinence. TVT. 
$\mathrm{E}$ xisten en la literatura médica más de 100 técnicas quirúrgicas descritas para la corrección de la incontinencia femenina de esfuerzo (IUE).

A mediados de los años 90 el Dr. Ulmsten et al. desarrollan el sistema TVT (Tension-free Vaginal Tape), basándose en la "teoría integral", según la cual la uretra se cierra en su porción media $^{1,2,3}$ La ausencia de soporte en uretra media a nivel de los ligamentos pubouretrales y en la pared anterior vaginal, así como el deterioro en la función e inserción de los músculos pubococcígeos, predispone a la incontinencia de esfuerzo. El TVT actuaría reemplazando estas deficientes estructuras, lo que ayudaría a restablecer la continencia. Este sistema consiste en una malla de polipropileno que se inserta por vía vaginal mediante una aguja especial, quedando ubicada en uretra media y autofijada sin necesidad de suturar ${ }^{4}$.

Dada la facilidad técnica de dicho sistema y los buenos resultados obtenidos en múltiples series, se ha impuesto en el tratamiento de la IUE.

El objetivo de este estudio es la revisión y exposición de los 100 primeros TVT implantados en nuestro servicio. Analizamos resultados y complicaciones obtenidas.

\section{MATERIAL Y METODOS}

Desde abril de 1999 a marzo de 2004 se han implantado en nuestro servicio 100 TVT a 100 pacientes con clínica de IUE. La edad media de las pacientes es de 56.5 años (R:30-80). Todas ellas presentan IUE aunque 54 (54\%) asocian urgencia miccional siendo predominante el componente de esfuerzo. Presentan IUE pura 46 (46\%) pacientes.

El seguimiento medio ha sido de 10.36 meses (R:1-54 meses) con revisión de resultados sobre la continencia de esfuerzo a los 6 meses de la intervención quirúrgica.

A todas las pacientes se les realiza historia clínica, exploración ginecológica y neurológica. En nuestro estudio se realiza EUD previo a cirugía fundamentalmente a aquellas pacientes que refieren IUE con urgencia asociada. En concreto al 92\% (50 pacientes) de las pacientes con IUE más urgencia (54 pacientes), y al $71 \%$ (33 pacientes) en aquellas que presentan IUE pura (46 pacientes).
La duración media de los síntomas antes de la consulta urológica es de 5.9 años (R:6 meses- 40 años). La intensidad de los síntomas la clasificamos en grado I (13 pacientes, 13\%), grado II (23 pacientes, 23\%) y grado III (26 pacientes, 26\%), definiendo como grado I los escapes de orina a grandes esfuerzos como tos/ estornudo, grado II moderados esfuerzos como correr/ recoger objetos del suelo/ subir escaleras, y grado III a pequeños esfuerzos como pequeños movimientos/ cambio de posición ${ }^{5,6}$.

Habían sido intervenidas previamente de IUE 12 pacientes $(12 \%)$, realizándose en 8 de ellas colposuspensión tipo Burch ( 8\%), en 2 plastia de Kelly ( $2 \%$ ), y en 2 técnica quirúrgica desconocida $(2 \%)$, siendo una de éstas últimas intervenida al mismo tiempo de cistocele.

De las 100 pacientes, 21 habían sufrido previamente cirugía de suelo pélvico: 13 histerectomía con doble anexectomía, 3 histerectomía simple, 2 intervenidas de prolapso cervical más cistocele, 1 cistoenterocele por vía vaginal, y 2 pacientes intervenidas de masas genitales ( quiste de Gardner y pólipos genitales).

Entre las 100 pacientes 62 presentaban cistocele, siendo grado I en 47 pacientes, grado II en 13 pacientes y grado III en 2 pacientes, definiendo grado I cuando la vejiga no alcanza el introito vaginal, grado II cuando llega al introito sin sobrepasarlo y grado III cuando pasa más allá del introito vaginal ${ }^{7}$. En 9 pacientes existe rectocele siendo 6 de ellas de grado I, y 3 de grado II. Dos pacientes presentan prolapso uterino.

Se realiza cirugía asociada al TVT en 17 casos, siendo en 15 de ellos cistorrafia ( 7 colocando malla), en un caso cirugía correctora del rectocele y en un caso cirugía compleja del suelo pélvico que comprende TVT más histerectomía vaginal más cistorrafia.

La cirugía se realiza bajo anestesia raquídea en 92 pacientes (92\%), anestesia local en 7 (7\%) y general en una (1\%). La paciente se coloca en posición de litotomía, realizando cateterismo vesical con sonda del 16 . Se realiza colporrafia anterior a nivel de uretra media y a $1 \mathrm{~cm}$. aproximadamente del meato uretral. A continuación se realizan 2 incisiones de $0.5 \mathrm{~cm}$. por encima de la sínfisis del pubis. Se procede a la disección parauretral bilateral; se tutoriza la sonda vesical 
con guía rígida lateralizando vejiga y uretra hacia el lado contrario del que estemos pasando la aguja, con el fin de no lesionar estas estructuras. Paso de la aguja: se conecta la aguja al introductor, y con ayuda de éste la aguja pasa parauretralmente perforando la fascia endopélvica hacia espacio retropúbico. Se desliza la aguja en íntimo contacto con la pared posterior del hueso púbico, se atraviesa el músculo recto del abdomen y piel. Repetimos la maniobra contralateralmente. Se realiza cistoscopia para comprobar la ausencia de lesión vesical. Posteriormente traccionamos de las agujas hasta situar la malla bajo la uretra media. Introducimos una tijera de Mayo entre la malla y uretra para conseguir la ausencia de tensión. Tras la colocación de la malla se retira la funda de plástico quedando fija gracias a la estructura del enrejado de polipropileno. Se corta la malla a ras de la piel abdominal, y colocamos grapas en estas incisiones. Por último suturamos la vagina, dejamos sonda vesical y taponamiento vaginal, retirándose ambos a las 24 horas de la intervención quirúrgica, midiendo residuo postmiccional, que si es $<150$ se da de alta a la paciente; con cifras $>150$ se realizan cateterismos intermitentes hasta conseguir residuos $<150^{5-9}$.

Hemos establecido como criterio de curación la comprobación objetiva de ausencia de fuga de orina por parte del médico junto con la referencia subjetiva de la paciente. Consideramos mejoría si la paciente refiere escapes ocasionales y mejoría subjetiva de su clínica previa. Fracaso si persiste el mismo grado de incontinencia que previo a la cirugía, o incluso empeoramiento.

\section{RESULTADOS}

Se han realizado 100 implantes de TVT en nuestro servicio. De estas pacientes 46 (46\%) presentaban IUE y 54 (54\%) asociaban urgencia a la IUE con predominio de ésta última. Se realiza estudio urodinámico (EUD) previo a cirugía a 83 pacientes (83\%), con resultado de IUE en 58 pacientes (58\%), IUM en 17 (17\%), y normal (no se objetivan fugas) en 8 (8\%).

Consideramos IUM tras EUD a aquellas mujeres con IUE más hiperactividad del detrusor.

De las pacientes con IUE pura (46), 7 (15,22\%) reciben tratamiento anticolinérgico previo a cirugía sin resultado satisfactorio. En el grupo de IUM
(54), se tratan un $45.59 \%$ (23 pacientes), de igual forma sin resultado satisfactorio.

La estancia media postoperatoria es de 1.24 días ( $\mathrm{R}: 1-13$ días).

Valoramos los resultados obtenidos a los 6 meses de la intervención quirúrgica: la tasa de curación es de un 65\% (65 pacientes), mejoría del $17 \%$ (17 pacientes), mientras que un $7 \%$ (7 pacientes) persisten con igual incontinencia. Un $11 \%$ (11 pacientes) se pierden durante el seguimiento (Fig. 1).

Hemos presentado un $12 \%$ de complicaciones (Fig. 2). En 3 pacientes (3\%) se produjo perforación vesical (2 intraoperatorias y 1 diferida). De ellas, 2 se reparan "in situ", manteniendo con sonda vesical hasta el momento del alta, en concreto 4 y 3 días tras la cirugía, realizándose en un paciente CUMS previo al alta, sin objetivar fugas. Una de ellas estaba intervenida previamente de IUE, y además, en el mismo acto quirúrgico de implantación de TVT se realiza corrección de cis-

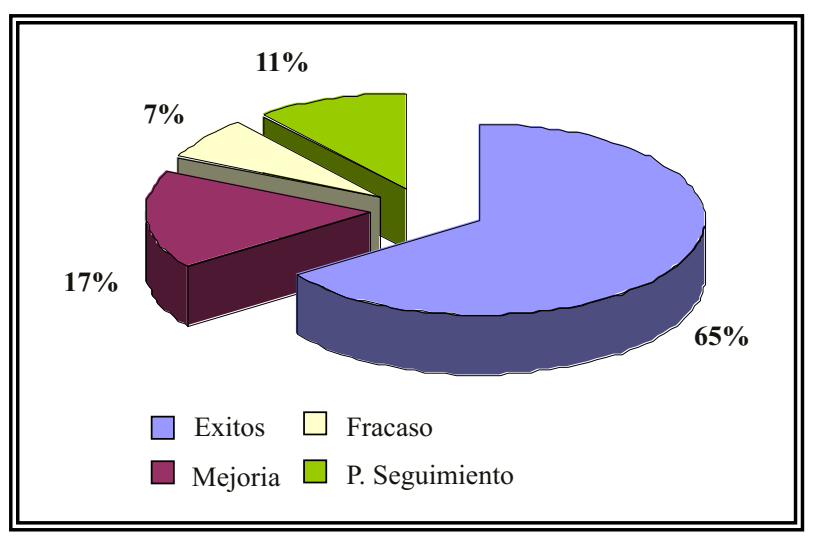

FIGURA 1

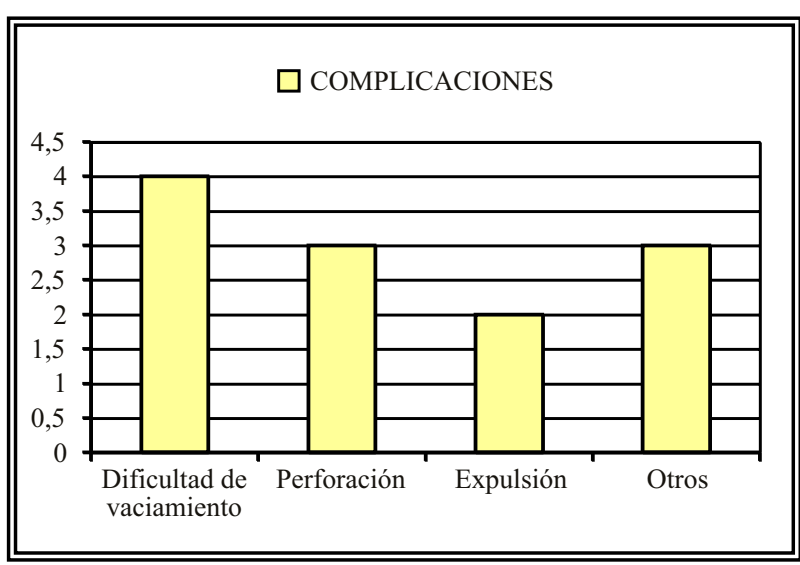

FIGURA 2 
tocele. En el tercer caso la paciente acudió 1 semana tras la intervención al Servicio de Urgencias con clínica de ITU e infección de herida quirúrgica y objetivando fístula urinaria, realizándose cistoscopia en la que se observa unos $0.5 \mathrm{~cm}$. de malla en cúpula vesical. Tras 5 días con sonda vesical se realiza CUMS con ausencia de fuga, retirando la sonda y siendo dada de alta.

Nos encontramos residuos altos en 3 pacientes (3\%), desapareciendo con autosondajes entre las 48 y 72 horas. Un paciente presenta en el postoperatorio inmediato RAO de repetición $\mathrm{y}$ micción con residuos altos por lo que se da el alta con cistostomía suprapúbica, retirándose a los 3 meses sin residuo y continente. Esta paciente se había sometido previamente a otra cirugía de incontinencia.

En 2 casos existe expulsión/ rechazo de bandeleta, uno de ellos presenta sangrado entre micciones y dispareunia de 7 meses de evolución, y la otra urgencia más dispareunia durante 2 meses. En ambos casos se realiza uretrolisis, presentando actualmente uno de ellos IUE grado I y la otra continencia completa. En una de ellas se había realizado cistorrafia junto a implantación de TVT.

En un paciente se coloca durante el acto quirúrgico talla vesical y a la retirada de la misma sufre un cuadro de peritonitis química por extravasación de orina. Se realiza un TAC abdominal observando como hallazgo casual tumor renal izquierdo, del que se interviene quirúrgicamente 2 meses después de la cirugía de IUE. Actualmente la paciente no presenta incontinencia aunque sí urgencia y nicturia ocasional.

Un caso presenta hematoma hipogástrico que se resuelve con medidas conservadoras, y un paciente molestias pélvicas subcrónicas con resolución espontánea (Tabla 1).

No tenemos ningún caso de RAO en postoperatorio tardío, de lesión uretral ni de infección de malla.

No hemos hecho constar en nuestro estudio el número de casos de ITUS postoperatorias, ya que en las pacientes intervenidas de TVT es frecuente la presencia de disuria y sintomatología miccional en el postoperatorio inmediato. Por este motivo no se realiza en nuestro servicio de forma estandarizada Gram en orina/urocultivo.
Tabla 1

\begin{tabular}{lc}
\hline Complicaciones & $\begin{array}{c}\text { Pacientes } \\
\text { (n=100) }\end{array}$ \\
\hline Tasa global de complicaciones (\%) & $12(12 \%)$ \\
Dificultad de vaciamiento vesical & $4(4 \%)$ \\
$\bullet$ Residuo >150 & 3 \\
• RAO & 1 \\
Perforación Vesical & $3(3 \%)$ \\
• Intraoperatoria & 2 \\
• Diferida & 1 \\
Expulsión/Rechazo de bandeleta & $2(2 \%)$ \\
Hematoma hipogástrico & $1(1 \%)$ \\
Peritonitis química & $1(1 \%)$ \\
Molestias pélvicas subcrónicas & $1(1 \%)$ \\
\hline
\end{tabular}

De los 7 pacientes con fracaso de la técnica quirúrgica, 2 son sometidos a administración de inyectables submucosos (inyección parauretral de carbono pirolítico); en los 2 casos persiste la IUE, realizándose en uno colposuspensión tipo Burch con resultado de mínima incontinencia de esfuerzo más urgencia, y en el otro se realiza $\mathrm{RAZ}$ más malla, presentando tras retirada de sonda RAO que se soluciona tras sondaje vesical durante 1 mes. En una paciente se implanta neuromodulador sacro tras fracaso de TVT, ya que presentaba importante incontinencia urinaria por urgencia junto al mismo grado de IUE, estando continente actualmente. A dos pacientes se les somete a tratamiento anticolinérgico (los 2 presentaban urgencia de novo tras TVT junto al mismo grado de IUE), presentando mejoría clínica un único paciente. Se retira TVT en 1 paciente acusando mayor IUE que previo a cirugía. El último caso rechaza cualquier tratamiento.

Un total de 27 pacientes (27\%) presentan urgencia postquirúrgica siendo 9 de éstos urgencia de novo, mientras que 18 ya la presentaban previamente a la cirugía $(9 \%$ y $18 \%$ del total respectivamente). De las pacientes con urgencia de novo $8(88,89 \%)$ son tratadas con anticolinérgicos con buenos resultados en 5 de ellas $(62,5 \%)$ y persistiendo la urgencia en $3(37,5 \%)$. De los 18 pacientes que ya presentaban urgencia previa, 10 $(55,67 \%)$ son tratados con anticolinérgicos, con buenos resultados en 6 (60\%), malos en 2 (20\%), y 2 con resultado desconocido (20\%) (Tabla 2). 
Tabla 2

\begin{tabular}{|c|c|c|c|c|}
\hline & & $\begin{array}{c}\text { Tratamiento } \\
\text { anticolinérgico }\end{array}$ & Mejoría & $\begin{array}{l}\text { Persistencia de } \\
\text { Urgencia }\end{array}$ \\
\hline $\begin{array}{l}\text { Tasa Global de Urgencia } \\
\text { Postquirúrgica (\%) }\end{array}$ & $27(27 \%)$ & $18(66,67 \%)$ & $11(61,11 \%)$ & $5(27,78 \%)$ \\
\hline Urgencia de Novo & $9(9 \%)$ & $8(88,89 \%)$ & $5(62,5 \%)$ & $3(37,5 \%)$ \\
\hline Urgencia pre y post-quirúrgica & $18(18 \%)$ & $10(55,67 \%)$ & $6(60 \%)$ & $2(20 \%)$ \\
\hline
\end{tabular}

En la mayoría de los estudios realizados la tasa de curación se sitúa por encima del $80 \% 4,6,9,10,11$. En nuestra serie hemos obtenido una tasa de curación del 65\%, y una mejoría del 17\%. Nuestros peo-

Presentan cirugía previa de IUE 12 pacientes (12\%): colposuspensión tipo Burch en 8 pacientes $(8 \%)$, plastia tipo Kelly en 2 (2\%), y en otros 2 casos (2\%) técnicas de incontinencia en la que el paciente no aporta informes. De estas 12 pacientes, 6 (50\%) presentan curación de su IUE tras implantación de TVT, 4 (33,33\%) mejoría y 2 $(16,67 \%)$ igual incontinencia a la previa. Únicamente 2 (16,67\%) presentaron complicaciones tras implantación de TVT, siendo 1 caso de RAO en postoperatorio (continente actualmente tras talla vesical durante 3 meses) y en otro dislaceración vesical con reparación in situ (tras 4 días de sondaje vesical presenta mejoría de su IUE).

A 17 (17\%) pacientes se les somete a cirugía de suelo pélvico asociado a TVT, siendo cistorrafia en 15 de ellos $(88,24 \%) 7$ de los cuales se colocó malla para reforzar. En 1 caso (5,88\%) se corrige rectocele de grado II, y en otro (5,88\%) se realiza cirugía compleja del suelo pélvico, que consistió en corrección del cistocele más histerectomía vaginal más TVT. Presentan curación 10 pacientes $(58,02 \%)$, mejoría 5 pacientes $(29,41 \%)$ y 2 pacientes $(11,76 \%)$ persisten con la misma IUE. Presentan complicaciones 2 pacientes de los intervenidos (11,76\%), en un caso expulsión/ rechazo de la bandeleta con posterior uretrolisis, y en el otro dislaceración vesical con reparación "in situ".

\section{DISCUSION}

Existen en la literatura médica más de 100 técnicas quirúrgicas para corregir la incontinencia de esfuerzo. En los últimos tiempos una de estas técnicas, el TVT se ha impuesto para este fin, basándose en la "teoría integral"según la cual el cierre de la uretra se produce a nivel de su porción media ${ }^{1,2,3}$.

Con este estudio queremos transmitir nuestra experiencia y resultados obtenidos con los 100 primeros sistemas TVT colocados en nuestro servicio. res resultados en comparación con otras series podría deberse a criterios de curación impuestos, en los que, como hemos explicado anteriormente, si la paciente no se encontraba objetivamente continente y satisfecha subjetivamente, se encuadraba en el grupo de mejoría. Por otro lado, tenemos una tasa de fracasos del 7\%, resultados muy similares al resto de series ${ }^{4}$. Entre ellos, se realiza en un caso cirugía previa de IUE (Burch), en otro cistorrafia asociada al TVT, y un tercero cirugía previa de IUE (Burch) asociando a la implantación de TVT corrección de rectocele, sin poder asegurar que éstos sean los factores desencadenantes.

Tenemos un $12 \%$ de complicaciones, siendo la más frecuente la dificultad de vaciamiento vesical (4\%) seguida de la perforación vesical (3\%), situación inversa a la mayoría de series estudiadas $^{4,6,10,12,13,14}$. Generalmente la dificultad del vaciamiento vesical es un hecho transitorio y es el resultado de la excesiva tensión de la malla o por la colocación del TVT en el contexto de vejiga hipocontráctil, solucionándose normalmente con cateterismos intermitentes durante unos dias. Si existe obstrucción completa puede ser necesaria la retirada del TVT ${ }^{3,4,13,15}$. En algunos casos este problema podría deberse a la implantación del sistema cerca del cuello vesical ${ }^{14}$. Otros autores defienden un aumento de la incidencia de residuos altos en el caso de cirugía de suelo pélvico asociada al TVT $^{4}$. En ninguno de nuestros 4 casos se da ésta última circunstancia. Los residuos altos se solucionaron de forma satisfactoria tras autosondajes en un tiempo máximo de 72 horas, y en el caso de RAO se colocó cistostomía suprapúbica durante 3 meses sin necesidad de retirada de TVT estando continente actualmente. En el EUD previo a TVT de esta paciente se observa detrusor hipoactivo y micción con ayuda de prensa abdominal. 
Como hemos mencionado anteriormente la perforación vesical es la complicación más frecuente en la mayoría de las series publica$\operatorname{das}^{4,10,12}$. Una de las razones aludidas a su producción es la cirugía previa de incontinencia ${ }^{4,6}$. En nuestro estudio, de los 3 casos de perforación vesical uno estaba intervenido previamente de IUE y además en el mismo acto quirúrgico de implantación de TVT se realizó cistorrafia anterior.

Una complicación poco frecuente es el rechazo/expulsión de la bandeleta ${ }^{3}$, presentando en nuestra serie 2 casos que fueron subsidiarios de uretrolisis. También tenemos un caso de peritonitis química que se produjo por la salida de orina al peritoneo al retirar la talla vesical que se había colocado en el acto quirúrgico por criterio médico sin que se hubiera producido ninguna complicación intraoperatoria.

Presentamos un caso de hematoma hipogástrico, complicación relativamente frecuente en el postoperatorio $(2-3,3 \%)$, generalmente relacionado con sangrado venoso en el espacio retropúbico, y que normalmente se resuelven con tratamiento conservador, como en nuestro caso, aunque se han descrito en la literatura hematomas subsidiarios de laparotomía ${ }^{4,7,13}$.

Otras complicaciones menos frecuentes son RAO en postoperatorio tardío, lesión uretral e infección de malla, no presentes en nuestro estudio. Lesiones inusuales aunque descritas como consecuencia del paso de las agujas son lesión de grandes vasos, lesión intestinal y del nervio obturador $^{4,10,16,17}$.

Hemos obtenido una tasa de urgencia de novo del $9 \%$, con tratamiento anticolinérgico en un $88.89 \%$, presentando buenos resultados el $62,5 \%$ de los tratados, siendo cifras similares a las obtenidas en otros estudios ${ }^{4,6,7,9}$. Éste hecho podría deberse a la colocación del sistema TVT cercano al cuello vesical ${ }^{4}$. Por otro lado parece ser que existen alteraciones biológicas causadas por la malla de prolene, así la urgencia de novo se relacionaría con cambios en el metabolismo de colágeno parauretral y con esclerosis alrededor de la malla ${ }^{6,14}$. Se necesitan más estudios para confirmar esta última teoría.

Una cuestión frecuentemente planteada es si el sistema TVT es un método adecuado para la corrección de IUE en aquellas pacientes que ya han sido sometidas previamente a cirugía de incontinencia. En este sentido Ulmsten y otros autores presentan buenos resultados entre un $74 \%$ y $81 \%{ }^{2,7,18,19}$. En nuestro caso tenemos 12 pacientes en esta situación con buenos resultados en un $83.33 \%$ (50\% curación completa y $33,33 \%$ mejoría significativa), por lo que podríamos decir que se trata de un método eficaz para este fin. La recurrencia de la IUE puede atribuirse a defectos anatómicos y/o funcionales que la cirugía no ha sido capaz de corregir, y que intentaría solventar el sistema TVT al reforzar el mecanismo de soporte de los ligamentos pubouretrales durante el estrés ${ }^{18}$.Los actos quirúrgicos previos sobre el suelo pélvico pueden originar fibrosis en el espacio de Retzius distorsionando los planos anatómicos y aumentando el riesgo de lesión en el tracto urinario inferior. La tasa de complicaciones tras cirugía repetida de incontinencia oscila entre $0-22 \%{ }^{18,19}$. En nuestro caso tenemos 2 pacientes con complicaciones $(16,67 \%)$, siendo éstas dislaceración vesical y RAO respectivamente.

En un $17 \%$ de los casos realizamos cirugía del suelo pélvico asociado a TVT. Diversos estudios revisados defienden que los resultados referentes a curación de incontinencia son similares en aquellos pacientes con implantación de TVT solo así como en los que se asocia cirugía de suelo pélvico (en torno a un 90\% de éxito en ambos casos), si bien en estas últimas existe un mayor porcentaje de complicaciones tales como perforación vesical, dificultad de vaciamiento y aparición de urgencia de novo (globalmente en torno a un $15.6 \%)^{14}$. En nuestra experiencia presentamos resultados satisfactorios en un $87.43 \%$, con curación completa en $58.02 \%$. En cuanto a complicaciones tenemos 2 casos $(11,76 \%)$, siendo rechazo de la bandeleta en uno de ellos y lesión vesical en el otro.

\section{CONCLUSIONES}

El sistema TVT es una técnica quirúrgica eficaz en el tratamiento de la IUE, sencilla de realizar, de fácil aprendizaje, que requiere escaso tiempo quirúrgico y corta estancia postoperatoria con rápida recuperación de las pacientes. 
Aunque presenta complicaciones, el porcentaje de éstas es bajo, pudiendo afirmar que se trata de una técnica segura.

Por otro lado es un procedimiento seguro, efectivo y mínimamente invasivo para el tratamiento de IUE recurrente.

Puede realizarse simultáneamente con cirugía reparadora del suelo pélvico con buenos resultados.

Por todo ello creemos que es una técnica recomendada para el tratamiento de la IUE.

\section{REFERENCIAS}

1. Petros P, Ulmsten U. An integral theory of female urinary incontinence. Experimental and clinical considerations. Acta Obstet Gynecol Scand 1990;153:7-31.

2. Petros P, Ulmsten U. An integral theory an its method for the diagnosis and management of female urinary incontinence. Scand J Urol Nephrol 1993;7-31.

3. Fillol Crespo M. Incontinencia urinaria de esfuerzo. Tratado de Uroginecología. Incontinencia urinaria 2004;145-157.

4. Debodinance P, Delporte P, Engrand J.B, Boulogne M. Tension-free vaginal tape (TVT) in the treatment of urinary stress incontinence: 3 years experience involving 256 operations. European Journal of Obstetrics \& Gynecology and Reproductive Biology 2002;105:49-58.

5. Briones Mardones G, Jiménez Cidre M, Fernández Fernández E, Perales Cabanas L, Pozo Mengual B, Escudero Barrilero A, et al. Tratamiento de la incontinencia urinaria de esfuerzo femenina con el sistema TVT (Tension free vaginal tape): nuestra experiencia. Actas Urol Esp 2001; 25(6):423-429.

6. Jeffry L, Deval B, Birsan A, Soriano D, Darai E. Objective and subjective cure rates alter tension-free vaginal tape for treatment of urinary incontinence. Urology 2001;58:702706

7. Jiménez Calvo J, Hualde Alfaro A, Santiago González de Garibay, Pinós Paul M, Jiménez Aristu J, Montesino Semper, M.et al TVT. 3 años de experiencia. Actas Urol Esp 2004;28(1):13-20.

8. Jiménez Calvo J, Hualde Alfaro A, Santiago González De Garibay A, et al: TVT (cinta libre de tensión) nueva técnica quirúrgica para el tratamiento de la incontinencia urinaria de esfuerzo. Arch Esp de Urol 2000;53(1):9-13.
9. Carlin B, Klutke J, Klutke C. The tension-free vaginal tape procedure for the treatment of stress incontinence in the female patient. Urology 2000;56(Suppl 6A):28-31.

10. Bombieri L, Freeman R.M. Surgery for stress urinary incontinence. Current Obstetrics \& Gynaecology 2003;13: 287-293

11. Sevestre S, Ciofu C, Deval B, Traxer O, Amarenco G, Haab F.: Results of the tension-free vaginal tape technique in the elderly. European Urology 2003;44:128-131.

12. Dolan L, Hilton P. Surgical management of stress incontinence: Which technique when?. EAU Update Series 2003; 1:154-165.

13. Karram M, Segal J, Vassallo B, Kleeman S. Complications and untoward effects of the tension-free vaginal tape procedure. Obstetrics \& Gynecology 2003;101:929-32.

14. Raffi A, Paoleotti X, Haab F, Levardon M, Deval B. Tensionfree vaginal tape and associated procedures: a case control study. European Urology 2004;45:356-361.

15. Klutke C, Siegel S, Carlin B, Paszkiewicz E, Kirkemo A, Klutke J.: Urinary retention after tension-free vaginal tape procedure: incidence and treatment. Urology 2001;58: 697-701.

16. Leboeuf L, Mendez L, Gousse A. Small bowel obstruction associated with tension-free vaginal tape. Urology 2004;63: 1182.e11-1182.e13.

17. Peyrat L, Boutin JM, Bruyere F, Haillot O, Fakfak H, Lanson Y. Intestinal perforation as a complication of tensionfree vaginal tape procedure for urinary incontinence. Eur urol 2001;39:603-5.

18. Lo T, Horng S, Chang C, Huang H, Tseng L, Liang C. Tension-free vaginal tape procedure alter previous failure in incontinence surgery. Urology 2002;60:57-61.

19. Rardin C, Kohli N, Rosenblatt P, Miklos J, Moore R, Strohsnitter W. Tension-free vaginal tape: outcomes among women with primary versus recurrent stress urinary incontinence. Obstetrics \& Gynecology 2002;100: 893-897.

Dra. C. Quicios Dorado

Avda. Casablanca $\mathrm{n}^{\circ} 1$, portal $22^{\circ} \mathrm{B}$

28223 Pozuelo de Alarcón. Madrid

e-mail:cquicios@yahoo.es

(Trabajo 22 de octubre 2004) 